\title{
Mean First Passage Time and Stochastic Resonance in a Transcriptional Regulatory System with Non-Gaussian Noise
}

\author{
Yan-Mei Kang*, Xi Chen and Xu-Dong Lin \\ School of Mathematics and Statistics, Xi'an Jiaotong University, \\ Xi'an 710049, P. R. China \\ Ning Tan \\ School of Aerospace, Xi'an Jiaotong University, \\ Xi'an 710049, P. R. China \\ *ymkang@mail.xjtu.edu.cn
}

Received 13 August 2015

Accepted 8 November 2016

Published 27 January 2017

Communicated by Benjamin Lindner

\begin{abstract}
The mean first passage time (MFPT) in a phenomenological gene transcriptional regulatory model with non-Gaussian noise is analytically investigated based on the singular perturbation technique. The effect of the non-Gaussian noise on the phenomenon of stochastic resonance (SR) is then disclosed based on a new combination of adiabatic elimination and linear response approximation. Compared with the results in the Gaussian noise case, it is found that bounded non-Gaussian noise inhibits the transition between different concentrations of protein, while heavy-tailed non-Gaussian noise accelerates the transition. It is also found that the optimal noise intensity for SR in the heavy-tailed noise case is smaller, while the optimal noise intensity in the bounded noise case is larger. These observations can be explained by the heavy-tailed noise easing random transitions.
\end{abstract}

Keywords: Non-Gaussian colored noise; mean first passage time; stochastic resonance; singular perturbation technique.

\section{Introduction}

The first passage time (FPT) is defined as the time that a walker or a particle spends before crossing a given position for the first time [1, 2], and is a value that fluctuates

This is an Open Access article published by World Scientific Publishing Company. It is distributed under the terms of the Creative Commons Attribution 4.0 (CC-BY) License. Further distribution of this work is permitted, provided the original work is properly cited.

* Corresponding author. 
with every trial. The mean first passage time (MFPT), or the statistical mean of the FPT, is an important quantity in stochastic processes and statistical mechanics, with wide applications ranging from the lifespan of electron devices [3] and neural firing dynamics [4] to the spread of diseases [5] and stochastic resonance (SR) $[6,7]$.

In gene regulation models, the FPT is referred to as the time for the protein concentration to transition from a lower level to a higher level or vice versa. As an important quantity that characterizes gene transcriptional regulation, the MFPT has begun to attract more and more attention [8-10]. For example, Zheng et al. investigated the effect of the maximal transcriptional rate and the noise correlation on the MFPT in an auto-activating positive-feedback loop [9], and Qiu et al. disclosed the dependence of the FPT distribution on the type of unstable attractor crossed by stochastic trajectories on the separatrix [10]. In particular, we note that the fluctuations involved in the existing investigations are modeled by Gaussian noise. Nevertheless, growing evidence suggests that transport in biological and chemical processes tends to be an anomalous process of long range correlation or long term memory [11-14]. Thus, in this paper, we prefer to model the fluctuation in gene regulation with a more appropriate kind of noise, namely non-Gaussian noise [15-20]. Our purpose is to analytically deduce the MFPTs of a gene transcriptional regulatory system driven by non-Gaussian noise. As an application of the derived MFPTs, we further explore the effect of non-Gaussian noise on SR based on a new combination of adiabatic elimination $[6,21,22]$ and linear response approximation [23-28].

The paper is organized as follows. In Sec. 2, following a brief introduction to the model, the exact expressions for the MFPTs in the gene regulatory system are deduced based on the singular perturbation analysis. In Sec. 3, the effect of nonGaussian noise on SR in the gene transcriptional regulatory system is disclosed and discussed. Finally, conclusions are given in Sec. 4 .

\section{MFPT under Non-Gaussian Colored Noise}

We consider a simple regulatory model $[29,30]$ that captures the salient features of transcription factor dimerization, binding, and phosphorylation-dependent regulation of transcription. As shown in Fig. 1, a single transcriptional activator TF-A is taken as part of the pathway mediating the cellular response to a stimulus, and the transcription factor TF-A forms a homodimer by phosphorylation that can bind to responsive elements (TF-REs). The $t f$ - $a$ gene then incorporates one of these responsive elements so that the TF-A transcription is enhanced when homodimers bind to the elements.

Assume that the transcription rate of $t f$ - $a$ saturates hyperbolically with TF-A dimer concentration and that the concentration of the homodimer is proportional to the square of the TF-A monomer concentration [29, 30]. Then, using a non-Gaussian noise term $u(t)$ to model the abnormal fluctuation occurring in transcriptional regulation, we acquire a simplified stochastic model for the $\nu$ concentration of the 


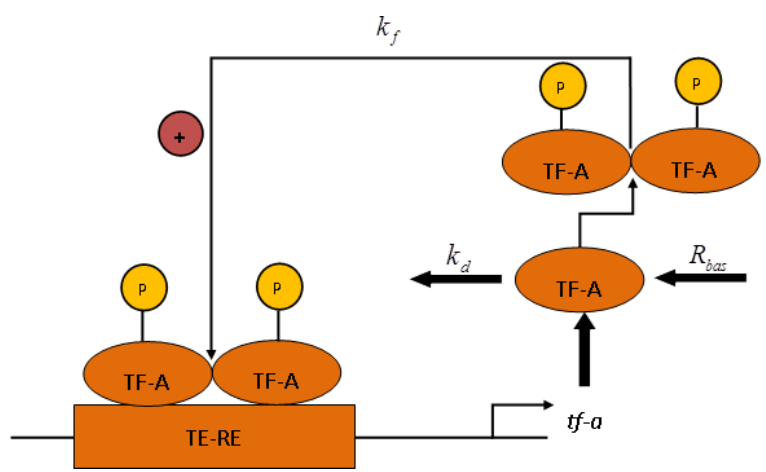

Fig. 1. Model of genetic regulation with a positive autoregulatory feedback loop. The transcription factor activator (TF-A) with rate $k_{f}$ when phosphorylated $(P)$ and binds as a dimer to specific responsive-element DNA sequence (TF-REs). TF-A is decomposed with rate $k_{d}$ and synthesized with rate $R_{\text {bas }}$.

TF-A monomer:

$$
\frac{d \nu}{d t}=\frac{k_{f} \nu^{2}}{\nu^{2}+K_{d}}-k_{d} \nu+R_{\text {bas }}+u(t),
$$

where $k_{f}$ is the maximal transcription rate of the $t f$ - $a$ gene, $K_{d}$ denotes the dissociation constant of the TF-A dimer from TF-Res, $k_{d}$ is a linear degradation constant, and $R_{\text {bas }}$ is the basal rate of synthesis at a negligible dimer concentration. The evolution of $u(t)$ is governed by the nonlinear Ornstein-Uhlenbeck process $[15,17]$

$$
\frac{d u(t)}{d t}=-\frac{1}{\tau_{0}} \frac{d V_{p}(u)}{d u}+\frac{1}{\tau_{0}} \varsigma(t)
$$

where $V_{p}(u)=D \operatorname{In}\left(1+\tau_{0}(p-1) u^{2} / 2 D /\left(\tau_{0}(p-1)\right)\right.$ with the extensive index $p$, the Gaussian white noise $\varsigma(t)$ satisfies $\langle\varsigma(t)\rangle=0$ and $\left\langle\varsigma(t) \varsigma\left(t^{\prime}\right)\right\rangle=2 D \delta\left(t-t^{\prime}\right)$, and the noise correlation time $\tau_{0}$ is a small parameter. According to $[15,19]$, the nonGaussian process governed by Eq. (2) has a stationary distribution for $p \in(-\infty, 3)$. In fact, when $p \in(1,3)$ the stationary distribution has a Tsallis-exponential asymptotic form, i.e., $P_{p}^{\text {as }}(u) \propto u^{-2 /(p-1)}$ as $|u| \rightarrow \infty$, and when $p \in(-\infty, 1)$, it is a cut-off distribution with critical value $u_{0}=\left[\frac{(1-p) \tau_{0}}{2 D}\right]^{-1 / 2}$. The linear Ornstein-Uhlenbeck process, namely the Gaussian colored noise process, can also be recovered from Eq. (2) when $p=1$. It is the fact that Eq. (2) covers bounded noise, Gaussian colored noise, and power-law heavy-tailed distributed noise when the extensive index $p \in(-\infty, 3)$ varies that motivates us to model the non-Gaussian fluctuation in such a way.

With linear stability analysis in mind, it is easy to find when the parameters satisfy

$$
\begin{gathered}
\left|K_{d}\left(k_{f}+R_{\text {bas }}\right) /\left(6 k_{d}\right)-\right|\left(k_{f}+R_{\text {bas }}\right) /\left.\left(3 k_{d}\right)\right|^{3}-K_{d} /\left.\left(2 k_{d}\right)\right|^{2} \\
+\left|K_{d} / 3-\right|\left(k_{f}+R_{\text {bas }}\right) /\left.\left.\left(3 k_{d}\right)\right|^{2}\right|^{3}<0,
\end{gathered}
$$




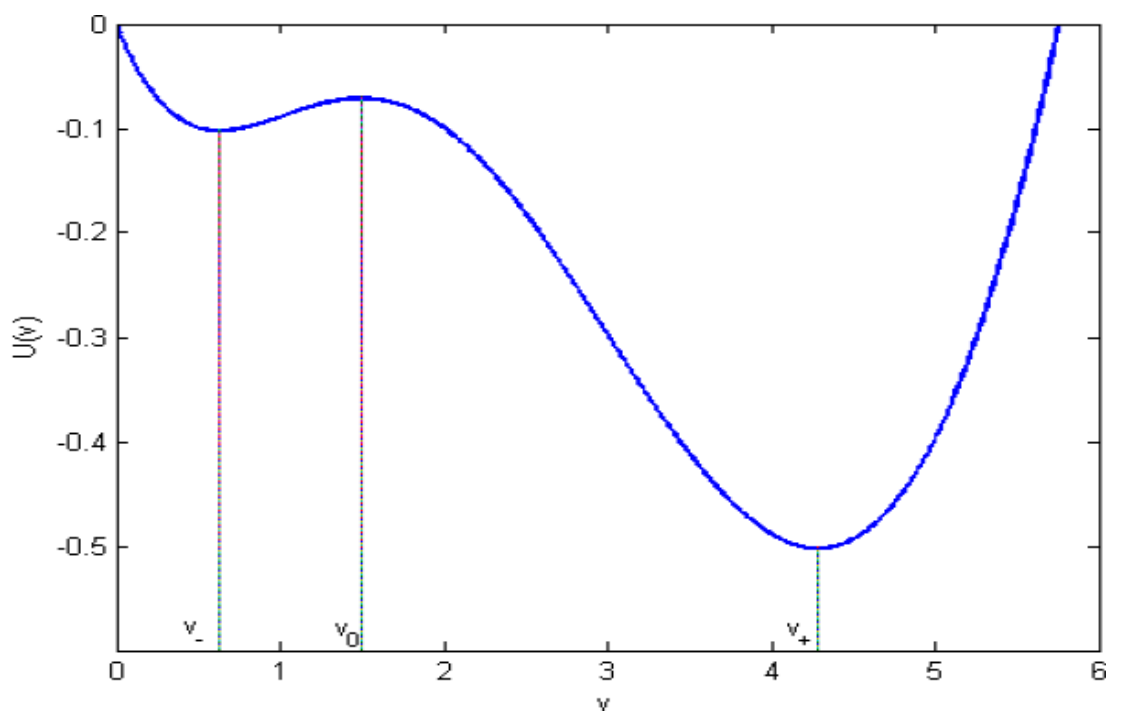

Fig. 2. The schema of the bistable potential $U(\nu)=-\int\left|k_{f} \nu^{2} /\left(v^{2}+K_{d}\right)-k_{d} v+R_{\text {bas }}\right| d \nu$ with $k_{f}=6, K_{d}=10, k_{d}=1, R_{\text {bas }}=0.4$. In this case, the stale equilibrium points are $v_{-} \approx 0.6268$ and $v_{+} \approx 4.2834$, and the unstable equilibrium point is $v_{0} \approx 1.4897$.

the deterministic counterpart of Eq. (1) has two stable equilibrium states that indicate different levels of protein concentration, namely

$$
\nu_{+}=2 \sqrt{p_{1} / 3} \cos \left(\theta_{1}\right)+\left(R_{\text {bas }}+k_{f}\right) /\left(3 k_{d}\right)
$$

and

$$
\nu_{-}=2 \sqrt{p_{1} / 3} \cos \left(\theta_{1}+2 \pi / 3\right)+\left(R_{\text {bas }}+k_{f}\right) /\left(3 k_{d}\right),
$$

and one unstable equilibrium state

$$
\nu_{0}=2 \sqrt{p_{1} / 3} \cos \left(\theta_{1}+4 \pi / 3\right)+\left(R_{\mathrm{bas}}+k_{f}\right) /\left(3 k_{d}\right),
$$

with

$$
\begin{aligned}
& p_{1}=\left[\left(R_{\text {bas }}+k_{f}\right) / k_{d}\right]^{2} / 3-K_{d}, \\
& q_{1}=K_{d}\left(k_{f}-2 R_{\text {bas }} /\left(3 k_{d}\right)-2\left[\left(R_{\text {bas }}+k_{f}\right) / k_{d}\right]^{3},\right.
\end{aligned}
$$

and

$$
\theta_{1}=\arccos \left(-q_{1} /\left(2 \sqrt{p_{1}^{3} / 27}\right) / 3\right) .
$$

Hence, in this case system (1) has two potential wells at $\nu_{+}$and $\nu_{-}$, separated by a potential barrier at $\nu_{0}$ when the noise level is low.

We now aim to deduce exact expressions for the MFPTs between different protein concentration states. Using the path integral technique [15], we can use the 
approximation $\frac{1}{\tau_{0}} \frac{d V_{p} u}{d u} \approx \frac{u}{\tau_{1}}$ with $\tau_{1}=\frac{2(2-p)}{(5-3 p)} \tau_{0}$ when $|p-1| \ll 1$ to rewrite Eq. (2) as

$$
\frac{d u(t)}{d t}=-\frac{1}{\tau_{1}} u(t)+\frac{1}{\tau_{1}} \xi(t),
$$

where the Gaussian white noise $\xi(t)$ satisfies $\langle\xi(t)\rangle=0$ and $\left\langle\xi(t) \xi\left(t^{\prime}\right)\right\rangle=2 D_{1} \delta\left(t-t^{\prime}\right)$ with $D_{1}=(2(2-p) /(5-3 p))^{2} D$. Substituting of Eq. (3) into Eq. (1) yields

$$
\left\{\begin{array}{l}
\frac{d \nu}{d t}=f(\nu)+\varepsilon^{-1} \sqrt{D_{1}} z(t) \\
\frac{d z}{d t}=-\varepsilon^{-2} z+\sqrt{2} \varepsilon^{-1} \xi(t),
\end{array}\right.
$$

where $\tau_{1}=\varepsilon^{2}$ and $f(v)=U^{\prime}(\nu)$. According to singular perturbation analysis [31-33], we can take $\varepsilon$ to be a small parameter. Let $P(\nu, z, t)$ be the solution of the Fokker-Planck equation

$$
\partial_{t} P(\nu, z, t)=\left[\varepsilon^{-2} \partial_{z}\left(z+\partial_{z}\right)-\varepsilon^{-1} \sqrt{D_{1}} z \partial_{\nu}-\partial_{\nu} f(\nu)\right] P(\nu, z, t),
$$

under an initial condition $\left.P(\nu, z, t)\right|_{t=0}=\delta\left(\nu-\nu_{-}\right) \mu(z)$ with $\mu(z)=e^{-z^{2} / 2} / \sqrt{2 \pi}$ and a half-absorbing boundary condition [31-33] at $\nu=\nu_{0}$. Then, the survival probability for a protein molecule that starts from $\nu=\nu_{-}$at $t_{0}=0$ and still stays in the left well at time $t$ reads $S\left(t \mid \nu_{-}\right)=\int_{0}^{\nu_{0}} \int_{-\infty}^{+\infty} P(\nu, z, t) d z d \nu$. As a result, the MFPT for the molecule moving from $v_{-}$to $v_{0}$ can be defined as $\left\langle T_{-}\right\rangle=\int_{0}^{\nu_{0}} \int_{-\infty}^{+\infty} \int_{0}^{+\infty} P(\nu, z, t) d t d z d \nu$. With $G(\nu, z)=\int_{0}^{+\infty} P(\nu, z, t) d t$ denoting the mean residence time at a point $(\nu, z)$ before crossing the potential barrier at $\nu=\nu_{0}[33]$, the MFPT can be rewritten as

$$
\left\langle T_{-}\right\rangle=\int_{0}^{\nu_{0}} \int_{-\infty}^{+\infty} G(\nu, z) d z d \nu .
$$

Integrating both sides of Eq. (5) with respect to $t$ from 0 to $+\infty$ leads to

$$
\left(\varepsilon^{-2} L_{0}+\varepsilon^{-1} L_{1}+L_{2}\right) G(\nu, z)=-\delta\left(\nu-\nu_{-}\right) \mu(z),
$$

where the differential operators are defined by $L_{0}=\partial\left(z+\partial_{z}\right), L_{1}=-\sqrt{D_{1}} z \partial_{\nu}$ and $L_{2}=-\partial_{\nu} f(\nu)$. If we seek a solution for Eq. (7) in the ansatz

$$
G(\nu, z)=G_{0}(\nu, z)+\varepsilon G_{1}(\nu, z)+\varepsilon^{2} G_{2}(\nu, z)+\cdots,
$$

then substitution of the ansatz into Eq. (7) yields a set of embedded equations:

$$
\begin{aligned}
& L_{0} G_{0}=0, \\
& L_{0} G_{1}=-L_{1} G_{0}, \\
& L_{0} G_{2}=-L_{1} G_{1}-L_{2} G_{0}-\delta\left(\nu-\nu_{1}\right) \mu(z), \\
& L_{0} G_{3}=-L_{1} G_{2}-L_{2} G_{1} .
\end{aligned}
$$

The embedded system can be iteratively solved by means of the integrability condition and the Hermite functions. 
Let $\rho_{n}(z)=e^{-z^{2} / 2} / \sqrt{2 \pi} \operatorname{He}_{n}(z)$ denote the $n$th Hermite function, with $\operatorname{He}_{n}(z)=$ $(-1)^{n} e^{z^{2} / 2}\left(\frac{d^{n}}{d z^{n}} e^{-z^{2} / 2}\right)$ Then $\rho_{n}(z)$ satisfies

$$
L_{0} \rho_{n}(z)=-n \rho_{n}(z)
$$

and the recurrence relation $z \rho_{n}(z)=\rho_{n+1}(z)+n \rho_{n-1}(z)$. Considering that $G_{0}(\nu, z)$ belongs to the null space of the operator $L_{0}$, we substitute $G_{0}(\nu, z)=\rho_{0}(z) r_{0}(\nu)$ into Eq. (9b) to obtain

$$
L_{0} G_{1}=\sqrt{D_{1}} \rho_{1}(z) \partial_{v} r_{0}(\nu) .
$$

With Eqs. (10) and (11) in mind, by setting $G_{1}(\nu, z)=\rho_{0}(z) r_{1}(\nu)-$ $\sqrt{D_{1}} \rho_{1}(z) \partial_{\nu} r_{0}(\nu)$ and repeating the above deduction, we obtain

$$
\begin{aligned}
L_{0} G_{2}= & \rho_{0}(z)\left(-\delta\left(\nu-\nu_{-}\right)-D_{1} \partial_{v}^{2} r_{0}(\nu)+\partial_{\nu} f(\nu) r_{0}(\nu)\right) \\
& +\sqrt{D_{1}} \rho_{1}(z) \partial_{v} r_{1}(\nu)-D_{1} \rho_{2}(z) \partial_{v}^{2} r_{0}(\nu)
\end{aligned}
$$

Because $L_{0}$ is only reversible in the subspace spanned by $\rho(z)(n \geq 1)$, the coefficient of $\rho_{0}(z)$ in Eq. (12) must vanish, which leads to

$$
-D_{1} \partial_{v}^{2} r_{0}(\nu)+\partial_{\nu} f(\nu) r_{0}(\nu)=\delta\left(\nu-\nu_{-}\right)
$$

For $\nu_{-}<\nu \leq \nu_{0}$, integration of Eq. (13) from $\nu$ to $\nu_{0}$ gives

$$
D_{1} \partial_{v} r_{0}(\nu)-f(\nu) r_{0}(\nu)=C_{1},
$$

where $C_{1}$ is the constant of integration. We further integrate Eq. (14) to obtain

$$
r_{0}(\nu)=\left(A_{1}-\frac{C_{1}}{D_{1}} \int_{v}^{\nu_{0}} \exp \left(-\frac{1}{D_{1}} \int_{u}^{v_{0}} f\left(\nu^{\prime}\right) d \nu^{\prime}\right) d u\right) \exp \left(-\frac{1}{D_{1}} \int_{\nu}^{\nu_{0}} f\left(\nu^{\prime}\right) d \nu^{\prime}\right) .
$$

The constant of integration $A_{1}$ is found to be zero from the absorbing boundary condition $r_{0}\left(\nu_{0}\right)=0$, and thus $r_{0}(\nu)$ can be reduced to

$$
r_{0}(\nu)=-\frac{C_{1}}{D_{1}} \int_{\nu}^{\nu_{0}} \exp \left(-\frac{1}{D_{1}} \int_{u}^{\nu_{0}} f\left(\nu^{\prime}\right) d \nu^{\prime}\right) d u \exp \left(-\frac{1}{D_{1}} \int_{\nu}^{\nu_{0}} f\left(\nu^{\prime}\right) d \nu^{\prime}\right) .
$$

Similarly, for $\nu<\nu_{-}$integrating Eq. (13) twice over 0 to $\nu$ gives

$$
r_{0}(\nu)=\left[\frac{C_{1}}{D_{1}} \int_{0}^{\nu} \exp \left(-\frac{1}{D_{1}} \int_{0}^{u} f\left(\nu^{\prime}\right) d \nu^{\prime}\right) d u+A_{2}\right] \exp \left(\frac{1}{D_{1}} \int_{0}^{\nu} f\left(\nu^{\prime}\right) d \nu^{\prime}\right) .
$$

Note that the kinetic term $f(\nu)$ in Eq. (4) satisfies $f(0)>0$, which means that the deterministic dynamics cannot drive the concentration of the TF-A monomer from the nonnegative range. Certainly, it is equally desirable that the weak noise should not make the concentration negative. As a result, we have to impose an absorbing boundary at $\nu=0$ to guarantee the nonnegativity of the concentration [34]. Because the absorbing boundary directly leads to $A_{2}=0$, we have

$$
r_{0}(\nu)=\frac{C_{2}}{D_{1}} \int_{0}^{\nu} \exp \left(-\frac{1}{D_{1}} \int_{0}^{u} f\left(\nu^{\prime}\right) d \nu^{\prime}\right) d u \exp \left(\frac{1}{D_{1}} \int_{0}^{\nu} f\left(\nu^{\prime}\right) d \nu^{\prime}\right) .
$$


According to Eq. (13), the continuity of $r_{0}(\nu)$ holds at $\nu=\nu_{-}$, namely $r_{0}\left(\nu_{-}\right)=$ $r_{0}\left(\nu_{-}+\right)$, which means that

$$
\begin{aligned}
& -C_{1} \int_{\nu_{-}}^{\nu_{0}} \exp \left(-\frac{1}{D_{1}} \int_{u}^{\nu_{0}} f\left(\nu^{\prime}\right) d \nu^{\prime}\right) d u \\
& \quad=C_{2} \int_{0}^{\nu_{-}} \exp \left(-\frac{1}{D_{1}} \int_{0}^{u} f\left(\nu^{\prime}\right) d \nu^{\prime}\right) d u \exp \left(\frac{1}{D_{1}} \int_{0}^{\nu_{0}} f\left(\nu^{\prime}\right) d \nu^{\prime}\right),
\end{aligned}
$$

and that the derivative $\partial_{\nu} r_{0}(\nu)$ has a jump discontinuity,

$$
D_{1} \partial_{\nu} r_{0}\left(\nu_{-}+\right)-D_{1} \partial_{\nu} r_{0}\left(\nu_{-}-\right)=-1
$$

which can be simplified to

$$
C_{1}-C_{2}=-1
$$

Simultaneously solving Eqs. (17) and (18) yields

$$
C_{1}=\frac{-\int_{0}^{v_{-}} \exp \left(-\frac{1}{D_{1}} \int_{0}^{u} f\left(\nu^{\prime}\right) d \nu^{\prime}\right) d u \exp \left(\frac{1}{D_{1}} \int_{0}^{\nu_{0}} f\left(\nu^{\prime}\right) d \nu^{\prime}\right)}{C}
$$

and

$$
C_{2}=\frac{\int_{v_{-}}^{v_{0}} \exp \left(-\frac{1}{D_{1}} \int_{u}^{v_{0}} f\left(\nu^{\prime}\right) d \nu^{\prime}\right) d u}{C}
$$

with $C=\int_{\nu_{-}}^{\nu_{0}} \exp \left(-\frac{1}{D_{1}} \int_{u}^{\nu_{0}} f\left(\nu^{\prime}\right) d \nu^{\prime}\right) d u+\int_{0}^{\nu_{-}} \exp \left(-\frac{1}{D_{1}} \int_{0}^{u} f\left(\nu^{\prime}\right) d \nu^{\prime}\right) d u \times$ $\exp \left(\frac{1}{D_{1}} \int_{0}^{\nu_{0}} f\left(\nu^{\prime}\right) d \nu^{\prime}\right)$. We finally obtain $r_{0}(\nu)$ by substituting $C_{1}$ and $C_{2}$ into Eqs. (15) and (16).

Analogously, setting

$$
G_{2}(\nu, z)=\rho_{0}(z) r_{2}(\nu)-\sqrt{D_{1}} \rho_{1}(z) \partial_{\nu} r_{1}(\nu)+D_{1} \rho_{2}(z) \partial_{\nu}^{2} r_{0}(\nu) / 2
$$

and substituting $G_{1}(\nu, z)$ and $G_{2}(\nu, z)$ into Eq. (9d) yields

$$
\begin{aligned}
L_{0} G_{3}(\nu, z)= & \sqrt{D_{1}} z \partial_{\nu}\left(\rho_{0}(z) r_{2}(\nu)-\sqrt{D_{1}} \rho_{1}(z) \partial_{\nu} r_{1}(\nu)+D_{1} \rho_{2}(z) \partial_{\nu}^{2} r_{0}(\nu) / 2\right) \\
& +\partial_{\nu} f(\nu)\left(\rho_{0}(z) r_{1}(\nu)-\sqrt{D_{1}} \rho_{1}(z) \partial_{\nu} r_{0}(\nu)\right)
\end{aligned}
$$

and annihilation of the coefficient of $\rho_{0}(z)$ leads to

$$
-\partial_{\nu} f(\nu) r_{1}(\nu)+D_{1} \partial_{1}^{2} r_{1}(\nu)=0 .
$$

A direct integration of Eq. (20) yields $r_{1}(\nu)=B \exp \left(-U(\nu) / D_{1}\right)$, where the constant $B$ can be determined by the half boundary condition $r\left(\nu_{0}\right)=\varepsilon \sqrt{D_{1}} \lambda_{M} r^{\prime}\left(\nu_{0}\right)$ with $\lambda_{M}=-\zeta(1 / 2) \approx 1.460354$ [31-33], where $\zeta(\cdot)$ denotes the Riemann zeta 
function. Substitution of $r\left(\nu_{0}\right)=r_{0}\left(\nu_{0}\right)+\varepsilon r_{1}\left(\nu_{0}\right)+\cdots$ into the boundary condition immediately gives $B=\left(\lambda_{M} / \sqrt{D_{1}}\right) \exp \left(U\left(\nu_{0}\right) / D_{1}\right)$, and thus we have

$$
r_{1}(\nu)=\lambda_{M} / \sqrt{D_{1}} \exp \left(\left(U\left(\nu_{0}\right)-U(\nu)\right) / D_{1}\right) .
$$

With the above expressions for $r_{0}(\nu)$ and $r_{1}(\nu)$ available, the MFPT can be calculated as

$$
\begin{aligned}
\left\langle T_{-}\right\rangle= & \int_{0}^{\nu_{0}} r_{0}(\nu) d \nu+\varepsilon \int_{0}^{\nu_{0}} r_{1}(\nu) d \nu \\
= & \frac{C_{2}}{D_{1}} \int_{0}^{\nu_{-}} \exp \left(-\frac{U(\nu)}{D_{1}}\right) d \nu \int_{0}^{\nu} \exp \left(\frac{U(u)}{D_{1}}\right) d u \\
& -\frac{C_{1}}{D_{1}} \int_{\nu_{-}}^{\nu_{0}} \exp \left(-\frac{U(\nu)}{D_{1}}\right) d \nu \int_{\nu}^{\nu_{0}} \exp \left(\frac{U(u)}{D_{1}}\right) d u+\varepsilon \int_{0}^{\nu_{0}} r_{1}(\nu) d \nu .
\end{aligned}
$$

We emphasize that the result in Eq. (22) is exact in the sense of discarding the higher-order terms in $\varepsilon$. To avoid involved calculation of integrals, we can simplify the above results by the steepest descent method [1] as follows:

$$
\begin{aligned}
& \frac{C_{2}}{D_{1}} \int_{0}^{\nu_{-}} \exp \left(\frac{U(u)}{D_{1}}\right) d u \int_{u}^{\nu_{-}} \exp \left(-\frac{U(\nu)}{D_{1}}\right) d \nu \\
& \quad \approx C_{2} \pi \exp \left(\frac{U\left(\nu_{0}\right)-U\left(\nu_{-}\right)}{D_{1}}\right)\left|U^{\prime \prime}\left(\nu_{0}\right) U^{\prime \prime}\left(\nu_{-}\right)\right|^{-\frac{1}{2}} \\
& \frac{C_{1}}{D_{1}} \int_{\nu_{-}}^{\nu_{0}} \exp \left(\frac{U(u)}{D_{1}}\right) d u \int_{\nu_{-}}^{u} \exp \left(-\frac{U(\nu)}{D_{1}}\right) d \nu \\
& \quad \approx C_{1} \pi \exp \left(\frac{U\left(\nu_{0}\right)-U\left(\nu_{-}\right)}{D_{1}}\right)\left|U^{\prime \prime}\left(\nu_{0}\right) U^{\prime \prime}\left(\nu_{-}\right)\right|^{-\frac{1}{2}}, \\
& \varepsilon \int_{0}^{\nu_{0}} r_{1}(\nu) d \nu \approx \sqrt{2 \pi} \varepsilon \lambda_{M}\left|U^{\prime \prime}\left(\nu_{-}\right)\right|^{-\frac{1}{2}} \exp \left(\frac{U\left(\nu_{0}\right)-U\left(\nu_{-}\right)}{D_{1}}\right) .
\end{aligned}
$$

Now Eq. (22) becomes

$$
\begin{aligned}
\left\langle T_{-}\right\rangle= & \pi\left(\left|U^{\prime \prime}\left(\nu_{0}\right) U^{\prime \prime}\left(\nu_{-}\right)\right|\right)^{-\frac{1}{2}} \exp \left(\frac{U\left(\nu_{0}\right)-U\left(\nu_{-}\right)}{D_{1}}\right) \\
& +\sqrt{2 \pi} \varepsilon \lambda_{M}\left|U^{\prime \prime}\left(\nu_{-}\right)\right|^{-\frac{1}{2}} \exp \left(\frac{U\left(\nu_{0}\right)-U\left(\nu_{-}\right)}{D_{1}}\right) .
\end{aligned}
$$

Similarly, we can obtain the MFPT from $\nu_{+}$to $\nu_{0}$ :

$$
\begin{aligned}
\left\langle T_{+}\right\rangle= & \pi\left(\left|U^{\prime \prime}\left(\nu_{0}\right)\right| U^{\prime \prime}\left(\nu_{+}\right)\right)^{-\frac{1}{2}} \exp \left(\frac{U\left(\nu_{0}\right)-U\left(\nu_{+}\right)}{D_{1}}\right) \\
& +\sqrt{2 \pi} \varepsilon \lambda_{M}\left|U^{\prime \prime}\left(\nu_{+}\right)\right|^{-\frac{1}{2}} \exp \left(\frac{U\left(\nu_{0}\right)-U\left(\nu_{+}\right)}{D_{1}}\right) .
\end{aligned}
$$

The theoretical results based on Eqs. (23) and (24) are shown in Figs. 3 and 4. As seen from Fig. 3, there is a good agreement between the theoretical and the 


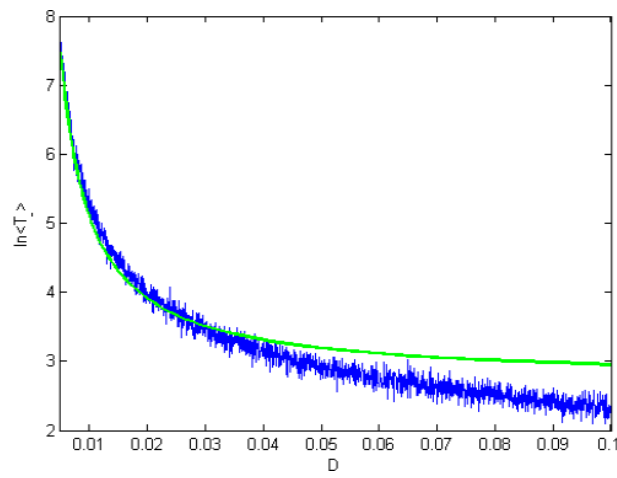

(a)

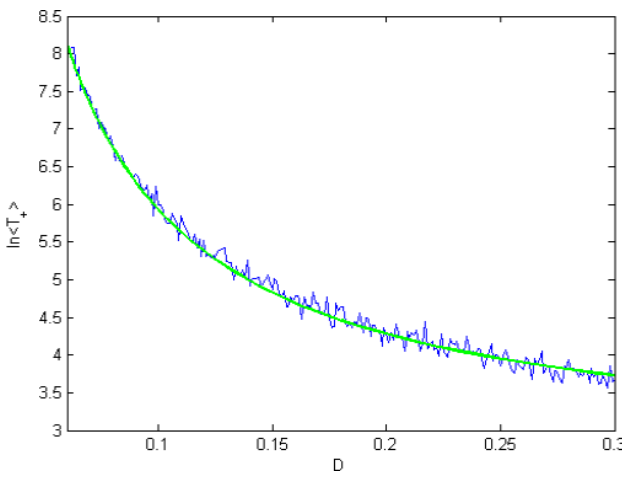

(b)

Fig. 3. Dependence of $\left\langle T_{-}\right\rangle$and $\left\langle T_{+}\right\rangle$on noise intensity with $\tau_{0}=0.1$ and $p=1.2$ : theoretical (green smooth) and simulated (blue coarse). The other parameters are same as Fig. 2.

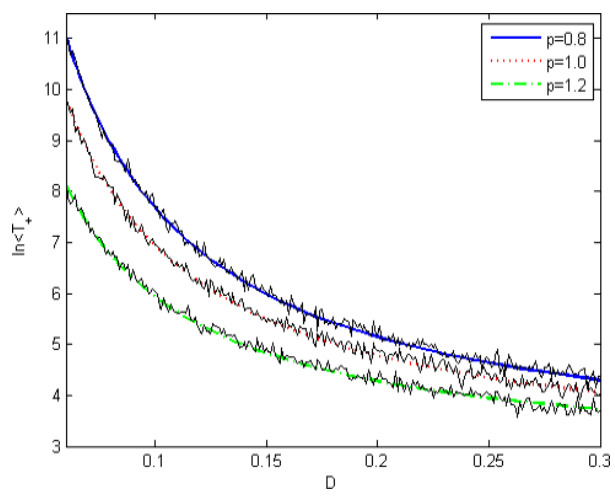

(a)
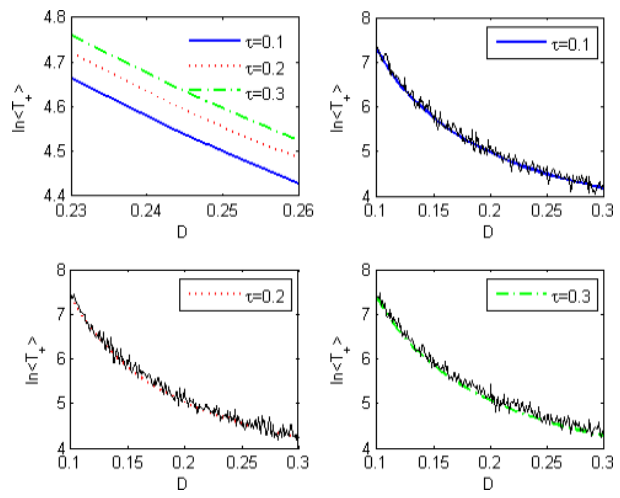

(b)

Fig. 4. Dependence of $\left\langle T_{+}\right\rangle$on the noise intensity $D$ with (a) different deviation parameters $p$ under $\tau_{0}=0.1$; (b) different correlation time $\tau_{0}$ under $p=0.9$. The smooth curves stand for theoretical results and coarse curves correspond to direct simulations. The other parameters are same as Fig. 2.

simulated results, but the discrepancy between them grows as the noise intensity increases, which is more obvious for $\left\langle T_{-}\right\rangle$than for $\left\langle T_{+}\right\rangle$. We remark that this discrepancy does not contradict the singular perturbation analysis, but rather it is a result of the steepest descent approximation, which can produce better accuracy for higher relative potential barriers (see Fig. 2). At the same time, we emphasize that the dependence of $\left\langle T_{-}\right\rangle$on the extensive index $p$ and the correlation time $\tau_{0}$ is similar to that of $\left\langle T_{+}\right\rangle$, so from Fig. 4 we can conclude that when $\tau_{0}$ and $D$ are fixed, the MFPT of the system under study is a decreasing function of the extensive index $p$, while with $p$ and $D$ fixed, the MFPT is a slowly increasing function of the 
correlation time $\tau_{0}$. Obviously, our observations generalize existing insights for the gene regulatory model.

\section{Effect of Non-Gaussian Noise on Stochastic Resonance}

According to $[35,36]$, an intrinsic cellular process can involve oscillation in the production of certain proteins, so the genetic circuits might also be modulated periodically by this protein. Based on this consideration, we introduce an external weak periodic signal $\kappa e^{-i \Omega t}$ of amplitude $\kappa$ and periodic $\Omega$ on the right-hand side of Eq. (1) to modulate gene regulation. As an application of the MFPTs derived above, in this section we investigate the effect of non-Gaussian noise on SR in the gene regulatory system.

Suppose that $\kappa \ll 1$ and that $\Omega \ll 1$. Then, the coherent signal can be approximated as constant in the sense of adiabatic elimination $[6,21,22]$, and thus Eqs. (23) and (24) can be modified into

$$
\begin{aligned}
\left\langle T_{-}\right\rangle= & \pi\left(\left|U^{\prime \prime}\left(\nu_{0}\right)\right| U^{\prime \prime}\left(\nu_{-}\right)\right)^{-\frac{1}{2}} \exp \left(\frac{\hat{U}\left(\nu_{0}, t\right)-\hat{U}\left(\nu_{-}, t\right)}{D_{1}}\right) \\
& +\sqrt{2 \pi} \varepsilon U^{\prime \prime}\left(\nu_{-}\right)^{-\frac{1}{2}} \lambda_{M} \exp \left(\frac{\hat{U}\left(\nu_{0}, t\right)-\hat{U}\left(\nu_{-}, t\right)}{D_{1}}\right)
\end{aligned}
$$

and

$$
\begin{aligned}
\left\langle T_{+}\right\rangle= & \pi\left(\left|U^{\prime \prime}\left(\nu_{0}\right)\right| U^{\prime \prime}\left(\nu_{+}\right)\right)^{-\frac{1}{2}} \exp \left(\frac{\hat{U}\left(\nu_{0}, t\right)-\hat{U}\left(\nu_{+}, t\right)}{D_{1}}\right) \\
& +\sqrt{2 \pi} \varepsilon U^{\prime \prime}\left(\nu_{+}\right)^{-\frac{1}{2}} \lambda_{M} \exp \left(\frac{\hat{U}\left(\nu_{0}, t\right)-\hat{U}\left(\nu_{+}, t\right)}{D_{1}}\right)
\end{aligned}
$$

with $\hat{U}(\nu, t)=-\int\left|k_{f} \nu^{2} /\left(\nu^{2}+K_{d}\right)-k_{d} \nu+R_{\text {bas }}+\kappa e^{-i \Omega t}\right| d \nu$.

To simplify the stochastic differential equation model to a two-state discrete model, let $A$ and $B$ represent the left and right potential wells. Noting that $R_{A B}=$ $1\left\langle T_{-}\right\rangle$and $R_{B A}=1\left\langle T_{+}\right\rangle$are the probability transition rates between the two stable states, we immediately obtain the two-state master equation

$$
\left\{\begin{array}{l}
\frac{d p_{A}(t)}{d t}=-R_{A B}(t) p_{A}(t)+R_{B A}(t) p_{B}(t) \\
\frac{d p_{B}(t)}{d t}=R_{A B}(t) p_{A}(t)-R_{B A}(t) p_{B}(t),
\end{array}\right.
$$

where $p_{A}$ and $p_{B}$ are the dwelling probabilities in the respective potential wells at time $t$.

Within a linear response range [25-28], we seek the long term solution of Eq. (27) in the form

$$
p_{A}^{(\mathrm{as})}(t)=p_{10}+p_{11} \kappa e^{-i \Omega t}, \quad p_{B}^{(\mathrm{as})}(t)=p_{20}+p_{21} \kappa e^{-i \Omega t},
$$


where $p_{i 0}$ and $p_{i 1}(i-1,2)$ are coefficients to be determined. Correspondingly, we set

$$
R_{A B}=R_{10}+R_{11} \kappa e^{-i \Omega t}, \quad R_{B A}=R_{20}+R_{21} \kappa e^{-i \Omega t}
$$

with

$$
\begin{aligned}
& R_{10}=\frac{1}{\pi\left(\left|U^{\prime \prime}\left(\nu_{0}\right)\right| U^{\prime \prime}\left(\nu_{-}\right)\right)^{-\frac{1}{2}}+\sqrt{2 \pi} \varepsilon U^{\prime \prime}\left(\nu_{-}\right)^{-\frac{1}{2}} \lambda_{M}} \exp \left(\frac{U\left(\nu_{-}\right)-U\left(\nu_{0}\right)}{D_{1}}\right), \\
& R_{20}=\frac{1}{\pi\left(\left|U^{\prime \prime}\left(\nu_{0}\right)\right| U^{\prime \prime}\left(\nu_{+}\right)\right)^{-\frac{1}{2}}+\sqrt{2 \pi} \varepsilon U^{\prime \prime}\left(\nu_{+}\right)^{-\frac{1}{2}} \lambda_{M}} \exp \left(\frac{U\left(\nu_{+}\right)-U\left(\nu_{0}\right)}{D_{1}}\right),
\end{aligned}
$$

$R_{11}=\frac{\left(\nu_{0}-\nu_{-}\right)}{D_{1}} R_{10}$ and $R_{21}=\frac{\left(\nu_{0}-\nu_{+}\right)}{D_{1}} R_{20}$. Substitution of Eqs. (28) and (29) into Eq. (27) yields

$$
\begin{aligned}
-\kappa i \Omega e^{-i \Omega t} p_{11}= & -\left(R_{10}+R_{11} \kappa e^{-i \Omega t}\right)\left(p_{10}+p_{11} \kappa e^{-i \Omega t}\right) \\
& +\left(R_{20}+R_{21} \kappa e^{-i \Omega t}\right)\left(p_{20}+p_{21} \kappa e^{-i \Omega t}\right), \\
-\kappa i \Omega e^{-i \Omega t} p_{21}= & -\left(R_{10}+R_{11} \kappa e^{-i \Omega t}\right)\left(p_{10}+p_{11} \kappa e^{-i \Omega t}\right) \\
& +\left(R_{20}+R_{21} \kappa e^{-i \Omega t}\right)\left(p_{20}+p_{21} \kappa e^{-i \Omega t}\right) .
\end{aligned}
$$

Comparison of the coefficients of $\kappa^{0}$ and $\kappa^{1}$ yields

$$
\begin{aligned}
& R_{10} p_{10}-R_{20} p_{20}=0, \\
& -i \Omega p_{11}=-R_{10} p_{11}+R_{20} p_{21}-R_{11} p_{10}+p_{20} R_{21} .
\end{aligned}
$$

With the requirement of conservation of probability in mind, namely

$$
p_{10}+p_{20}=1, \quad p_{11}+p_{21}=0,
$$

the solutions of Eqs. (31) and (32) can be written as

$$
p_{10}=\frac{R_{20}}{R_{10}+R_{20}}, \quad p_{20}=\frac{R_{10}}{R_{10}+R_{20}}
$$

and

$$
\begin{aligned}
& p_{11}=\frac{1}{R_{10}+R_{20}} \frac{R_{10} R_{21}-R_{11} R_{20}}{\left(R_{10}+R_{20}\right)-i \Omega}, \\
& p_{21}=\frac{1}{R_{10}+R_{20}} \frac{R_{11} R_{20}-R_{10} R_{21}}{\left(R_{10}+R_{20}\right)-i \Omega},
\end{aligned}
$$

respectively. Substituting Eqs. (33) and (34) into Eq. (28), we obtain the long term average of the output response of the two-state model as

$$
E X=\nu_{-} p_{10}+\nu_{+} p_{20}+\left(\nu_{-} p_{11}+\nu_{+} p_{21}\right) \kappa e^{-i \Omega t}
$$

and consequently the spectral amplification factor is

$$
|\chi|^{2}=\kappa^{-2}\left|\nu_{-} p_{11}+\nu_{+} p_{21}\right|^{2} .
$$

In Fig. 5, the nonmonotonic dependence of the SAF via the noise intensity signifies the occurrence of SR in the gene regulatory systems. From this figure, we see that 


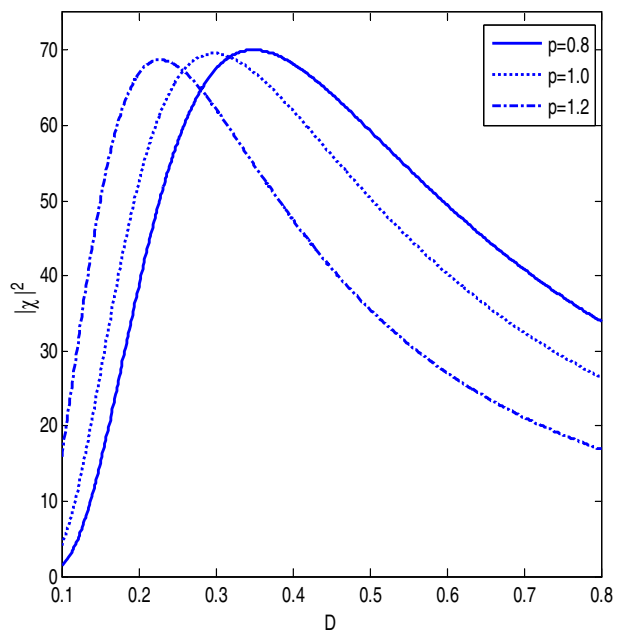

(a)

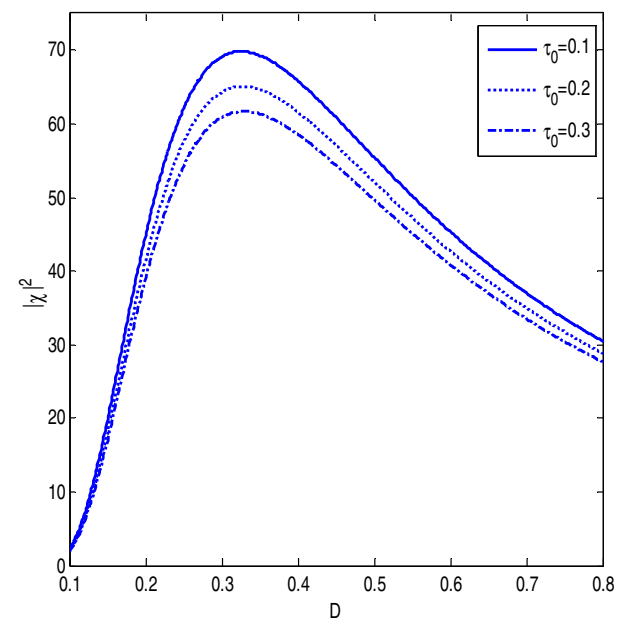

(b)

Fig. 5. Dependence of the spectral amplification factor on the noise intensity with $\Omega=0.1, \kappa=0.3$ and (a) $\tau_{0}=0.1$; (b) $p=0.9$. The other parameters are same as Fig. 2.

the peak SR is almost constant for different extensive indices, but that the optimal noise intensity lessens as the extensive index increases (Fig. 5(a)). We also observe that the resonant peak becomes weak when the noise correlation time is prolonged (Fig. 5(b)), and thus it can be concluded that the correlation time of the nonGaussian noise has the same tendency in its effect on SR as that of Gaussian colored noise [37, 38].

Theoretically, we have determined the effect of non-Gaussian noise on SR in the gene regulatory model from the viewpoint of two-state approximation. Nevertheless, because the probability transition rates used in the two-state model is an approximate outcome from the steepest descent method and especially the two-state model captures the nonlocal interwell random jumps but omits the local intrawell motion, we have to adopt stochastic simulation to check whether the above discussion on SR can characterize the real effect of non-Gaussian noise on SR in the original gene regulatory model. To clarify this point, we exhibit some typical time history in Fig. 6. From the picture, it is clear that there occur two different motions in the bistable system, at the weak noise level, the intrawell fluctuation is dominated, while on the strong noise level, the interwell jump is intertwisted with the intrawell motion. This suggests us that the negligence of the intrawell motion might cause certain quantitative deviations. Actually, we show the dependence of the spectral amplification factor on noise intensity in Fig. 7. Here, the spectral amplification factor is captured from the long time quasi-stationary dynamics which is obtained by discarding the transient response over the first several periods. By comparing Fig. 6 with Fig. 7, it is easy to observe evident quantitative differences in the resonant 

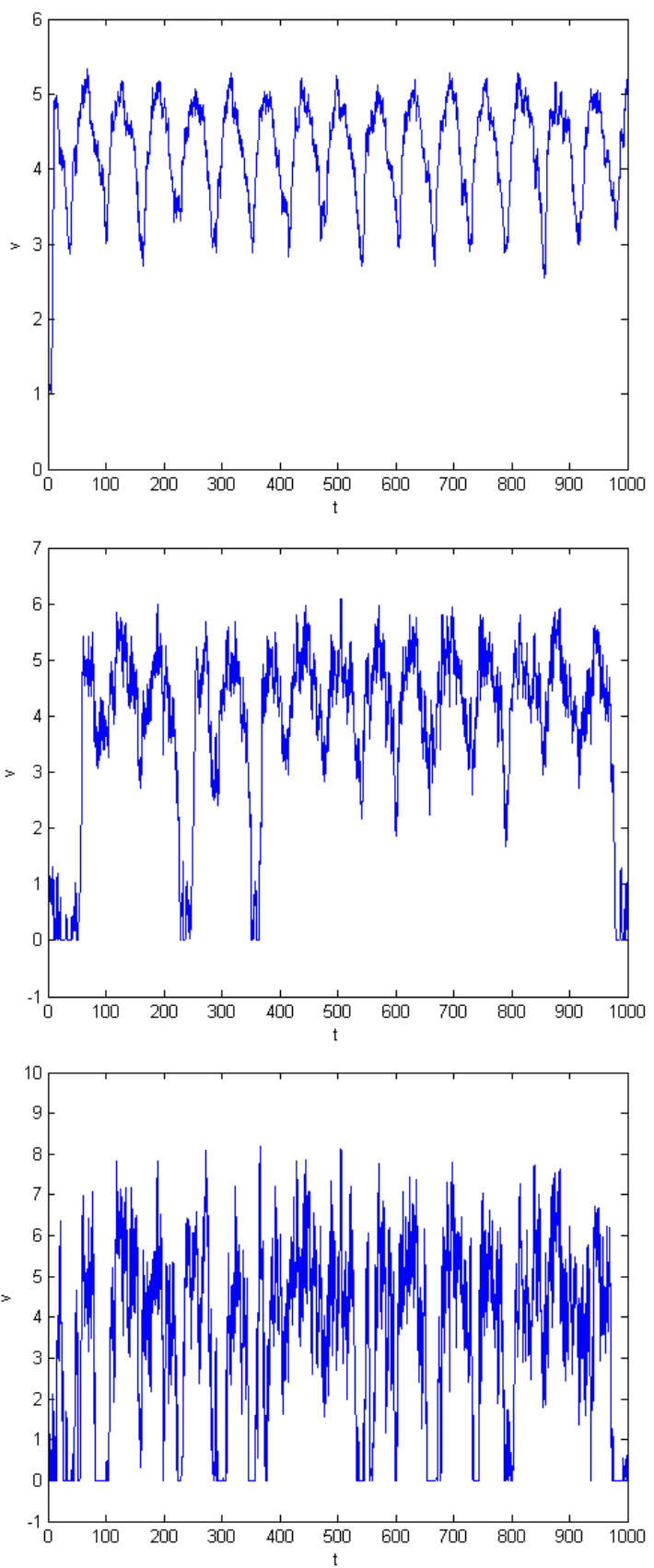

Fig. 6. The simulation on the evolution of the model (1) with weak periodic signal with $p=1$ and $\tau_{0}=0.1$. From the top to the bottom, the noise intensity denotes $D=0.1, D=0.5$ and $D=0.9$, respectively. The other parameters are same as Fig. 5. Here the nonnegativity of the concentration is guaranteed by imposing an absorbing boundary condition to the model (1), as treated in Sec. 2. That is to say, when the concentration variable is crossing zero from above, it will be reset to zero. 


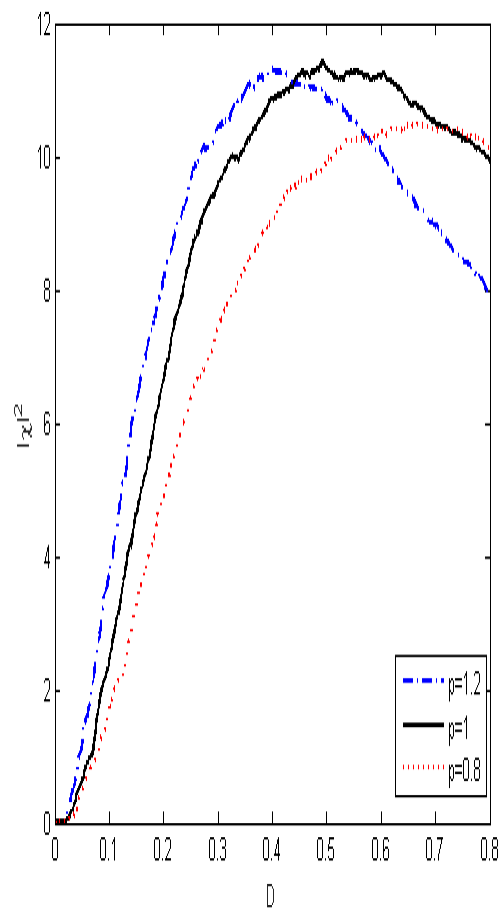

(a)

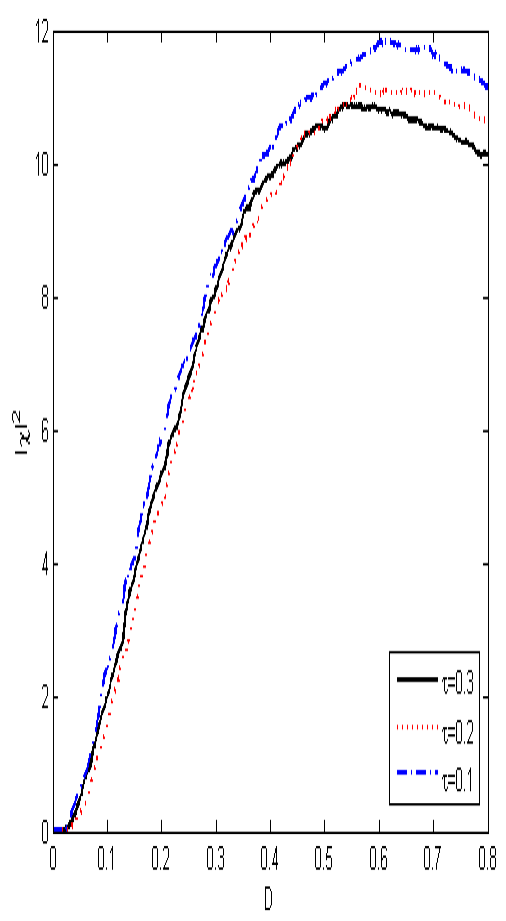

(b)

Fig. 7. Simulation for the dependence of the spectral amplification factor on the noise intensity with $\Omega=0.1, \kappa=0.3$ and (a) $\tau_{0}=0.1$; (b) $p=0.9$. The other parameters are same as Fig. 5 .

curves between the two methods. Fortunately, the effect of the extensive index and the correlation time of non-Gaussian noise on SR between the two methods is similar. That is, changing the extensive index almost only shifts the location of the resonant peak while increasing the correlation time weakens the resonant effects. Undoubtedly, this qualitative agreement confirms our theoretical observations to some extent.

\section{Discussion and Conclusion}

We have derived an explicit formula for the MFPT for a phenomenological gene transcriptional regulatory model with non-Gaussian colored noise using singular perturbation analysis and the steepest descent approximation. When the extensive index is close to 1 , we found that the MFPT is a decreasing function of the extensive index of the non-Gaussian noise. Noting that as the extensive index increases, the non-Gaussian noise changes from bounded noise $(p<1)$ to Gaussian colored noise $(p=1)$ and then heavy-tailed distribution noise $(p>1)$, we conclude from our investigation that heavy-tailed distribution noise is more helpful for protein concentration transition than bounded noise. This characteristic reflects the universal fact 
that the heavy-tailed distribution noise easily induces more large random jumps than bounded noise.

We have also demonstrated the phenomenon of SR in the gene regulatory model. Adiabatic elimination and linear response theory are two fundamental mathematical techniques established for SR, and we have deduced a connection between them for the first time. By altering the extensive index, it is found that the peak SR does not vary obviously as the extensive index increases, but that the optimal noise intensity for SR is a decreasing function of the index. Compared with the case of Gaussian colored noise, the optimal noise intensity is smaller in the case of heavytailed non-Gaussian noise, but larger in the case of bounded non-Gaussian noise. Because SR is a synchronization effect between a noise-induced switch and weak signal modulation in the time-modulated bistable model [39], the weaker optimal noise intensity for heavy-tailed distribution noise is most likely because it easily induces more random jumps.

Additionally, as with the time correlation of Gaussian noise [37, 38], our investigation demonstrates that the time correlation for heavy-tailed or bounded nonGaussian noise has an inhibitive effect on SR. Again, this observation depends on the ease of noise-induced random switches. The qualitative characteristic of these results has been confirmed by simulations and should be useful in gene circuit design.

\section{Acknowledgment}

The authors appreciate the support of Chinese Natural Science Foundation with Grant No. 11372233.

\section{References}

[1] C. W. Gardiner, Handbook of Stochastic Methods for Physics, Chemistry and the Natural Sciences (Springer-Verlag, Berlin, 1985).

[2] J. Q. Duan, An Introduction to Stochastic Dynamics (Science Press, Beijing, 2015).

[3] P. Talkner, Mean first passage time and the lifetime of a metastable state, Z. Phys. B-Condens. Matter 68 (1987) 201-207.

[4] T. Tetzlaff and G. T. Einevoll, Rate dynamics of leaky integrate-and-fire neurons with strong synapses, Front. Comput. Neurosci. 4 (2010) 149.

[5] V. Mendez, D. Campos and W. Horsthemke, Stochastic fluctuations of the transmission rate in the susceptible-infected-susceptible epidemic model, Phys. Rev. E $\mathbf{8 6}$ (2012) 011919.

[6] B. McNamara and K. Wiesenfeld, Theory of stochastic resonance, Phys. Rev. E 9 (1989) 4854.

[7] S. H. Li and J. C. Wu, Stochastic resonance in a bacterium growth system with time delay and colored noise, Fluct. Noise Lett. 14(2) (2015) 1550019.

[8] G. Vahedi and J. F. Chamberland, Intervention in gene regulatory networks via a stationary mean-first passage time control policy, IEEE Trans. Biol. Med. Eng. 55(10) (2008) 2319-2331. 
[9] X. D. Zheng, X. Q. Yang and Y. Tao, Bistability, probability transition rate and first passage time in an autoactivating positive-feedback loop, PLoS One 6(3) (2011) e17104.

[10] H. Qiu, Z. Yuan and T. Zhou, Distribution of the first passage time in bistable biological systems, Chin. J. Phys. 50(5) (2012) 857-867.

[11] K. Wiesenfeld, D. Pierson, E. Pantazelou, C. Dames and F. Moss, Stochastic resonance on a circle, Phys. Rev. Lett. 72 (1994) 2125-2129.

[12] D. Nozaki, D. J. Mar, P. Grigg and J. J. Collins, Effects of colored noise on stochastic resonance in sensory neurons, Phys. Rev. Lett. 82 (1999) 2402-2405.

[13] R. Metzler and J. Klafter, The random walk's guide to anomalous diffusion: A fractional dynamics approach, Phys. Rep. 339 (2000) 1-77.

[14] R. Metzler and J. Klafter, The restaurant at the end of the random walk: Recent developments in the description of anomalous transport by fractional dynamics, J. Phys. A, Math. Gen. 37 (2004) R161-R208.

[15] M. A. Fuentes, H. S. Wio and R. Toral, Effective Markovian approximation for nonGaussian noises: A path integral approach, Phys. A 303 (2002) 91-104.

[16] H. Hasegawa, A moment approach to non-Gaussian colored noise, Physica A $\mathbf{3 8 4}$ (2007) 241-258.

[17] H. Zhang, W. Xu and Y. Xu, The study on a stochastic system with non-Gaussian noise and Gaussian colored noise, Phys. A 388 (2009) 781-788.

[18] Y. Huang and X. B. Liu, Stochastic stability of viscoelastic system under nonGaussian colored noise excitation, Sci. Chin. Phys. Mech. Astron. 55 (2012) 483-492.

[19] C. Tsallis, Possible generalization of Boltzmann-Gibbs statistics, J. Stat. Phys. 52 (1988) 479-487.

[20] S. Bouzat and H. S. Wio, New aspects on current enhancement in Brownian motors driven by non-Gaussian noises, Phys. A 351 (2005) 69-78.

[21] G. Hu, Random Force and Nonlinear System (Shanghai Scientific Education Publishing House, Shanghai, 1994) (in Chinese).

[22] Y. M. Kang, J. X. Xu and Y. Xie, Stochastic resonance in two-dimensional Brownian motion in the weak noise limit, Acta Phys. Sin. 52(4) (2003) 802-807. (in Chinese).

[23] H. Risken, The Fokker-Planck Equation: Methods of Solution and Applications (Springer-Verlag, Berlin, New York, 1984).

[24] P. Jung, Periodically driven stochastic systems, Phys. Rep. 234 (1993) 175-295.

[25] Y. M. Kang, J. X. Xu and Y. Xie, Observing stochastic resonance in an underdamped bistable Duffing oscillator by the method of moments, Phys. Rev. E68 (2003) 036123.

[26] Y. M. Kang, Coherence resonance in subdiffusive fractional Klein-Kramers periodic potential systems without a bifurcation precursor, Europhys. Lett. 94 (2011) 60005.

[27] Y. M. Kang, Y. L. Jiang and Y. Xie, Linear response characteristics of time-dependent time fractional Fokker-Planck equation systems, J. Phys. A, Math. Theor. 47 (2014) 455005.

[28] J. Casado-Pascual, J. Gomez-ordonez M. Morillo and P. Hanggi, Checking linear response theory in driven bistable systems, Fluct. Noise Lett. 2 (2002) L127-L138.

[29] A. D. Keller, Model genetic circuits encoding autoregulatory transcription factors, J. Theor. Biol. 172 (1995) 169-185.

[30] P. Smolen, D. A. Baxter and J. H. Byrne, Frequency selectivity, multistability, and oscillations emerge from models of genetic regulatory systems, Cell Physiol. 274(2) (1998) C531-C542

[31] C. R. Doering, P. S. Hagan and C. D. Levermore, Bistability driven by weakly colored Gaussian noise: The Fokker-Planck boundary layer and mean first-passage times, Phys. Rev. Lett. 59 (1987) 2129-2132. 
[32] P. S. Hagan, C. R. Doering and C. D. Levermore, Mean exit times for particles driven by weakly colored noise, SIAM J. Appl. Math. 49 (1989) 1480-1513.

[33] D. Andrieux and T. Monnai, Firing rate of noisy integrate-and-fire neurons with synaptic current dynamics, Phys. Rev. E 80 (2009) 021933.

[34] W. Horsthemke and R. Lefever, Noise-induced Transitions: Theory and Applications in Physics, Chemistry and Biology (Springer-Verlag, Berlin, 1984).

[35] J. Hasty, F. Isaacs, M. Dolnik, D. McMillen and J. J. Collins, Designer gene networks: Towards fundamental celluler control, Chaos 11 (2001) 207-220.

[36] T. S. Zhou, Stochastic Dynamics in Biological Systems (Science Publishing House, Beijing, 2009).

[37] P. Hanggi, P. Jung, C. Zerbe and F. Moss, Can colored noise improve stochastic resonance? J. Stat. Phys. 70 (1993) 25-47.

[38] Y. M. Kang and Y. L. Jiang, A semi-analytic method for computing the long-time order parameter dynamics in mean-field coupled overdamped oscillators with colored noises, Phys. Lett. A 372 (2008) 6826-6832.

[39] L. Gammaitoni, P. Hänggi, P. Jung and F. Marchesoni, Stochastic resonance, Rev. Mod. Phys. 70(1) (1998) 223-287. 